OPEN ACCESS

Edited by:

Lei Deng,

Central South University, China

Reviewed by:

Yongchun Zuo,

Inner Mongolia University, China

Shaherin Basith,

Ajou University, South Korea

${ }^{*}$ Correspondence:

Hao Lin

hlin@uestc.edu.cn

Specialty section:

This article was submitted to

Molecular Medicine,

a section of the journal

Frontiers in Cell and Developmental

Biology

Received: 13 July 2020 Accepted: 15 September 2020

Published: 15 October 2020

Citation

Zhang Z-M, Wang J-S, Zulfiqar H, Lv H, Dao F-Y and Lin H (2020) Early

Diagnosis of Pancreatic Ductal

Adenocarcinoma by Combining Relative Expression Orderings With

Machine-Learning Method.

Front. Cell Dev. Biol. 8:582864.

doi: $10.3389 /$ fcell.2020.582864

\section{Early Diagnosis of Pancreatic Ductal Adenocarcinoma by Combining Relative Expression Orderings With Machine-Learning Method}

\author{
Zi-Mei Zhang, Jia-Shu Wang, Hasan Zulfiqar, Hao Lv, Fu-Ying Dao and Hao Lin*
}

Key Laboratory for Neuro-Information of Ministry of Education, Center for Informational Biology, School of Life Sciences and Technology, University of Electronic Science and Technology of China, Chengdu, China

Pancreatic ductal adenocarcinoma (PDAC) is an aggressive and lethal cancer deeply affecting human health. Diagnosing early-stage PDAC is the key point to PDAC patients' survival. However, the biomarkers for diagnosing early PDAC are inexact in most cases. Therefore, it is highly desirable to identify an effective PDAC diagnostic biomarker. In the current work, we designed a novel computational approach based on withinsample relative expression orderings (REOs). A feature selection technique called minimum redundancy maximum relevance was used to pick out optimal REOs. We then compared the performances of different classification algorithms for discriminating PDAC and its adjacent normal tissues from non-PDAC tissues. The support vector machine algorithm is the best one for identifying early PDAC diagnostic biomarker. At first, a signature composed of nine gene pairs was acquired from microarray gene expression data sets. These gene pairs could produce satisfactory classification accuracy up to $97.53 \%$ in fivefold cross-validation. Subsequently, two types of data from diverse platforms, namely, microarray and RNA-Seq, were used to validate this signature. For microarray data, all (100.00\%) of 115 PDAC tissues and all (100.00\%) of 31 PDAC adjacent normal tissues were correctly recognized as PDAC. In addition, $88.24 \%$ of 17 non-PDAC (normal or pancreatitis) tissues were correctly classified. For the RNA-Seq data, all (100.00\%) of 177 PDAC tissues and all (100.00\%) of 4 PDAC adjacent normal tissues were correctly recognized as PDAC. Validation results demonstrated that the signature had a good cross-platform effect for early detection of PDAC. This work developed a new robust signature that might be a promising biomarker for early PDAC diagnosis.

Keywords: pancreatic ductal adenocarcinoma, biomarker, relative expression orderings, diagnosis, support vector machine

\section{INTRODUCTION}

Pancreatic ductal adenocarcinoma (PDAC) is one of the deadliest malignant carcinomas and it accounts for at least $95 \%$ of all pancreatic cancer cases (Tanaka, 2016). PDAC has a poor survival outcome (Zhang et al., 2018b) by reason of the difficulty of diagnosing and assessing PDAC at an early stage. Most patients with PDAC do not present any 
specific early characteristics during the early stage, which means that early PDAC cannot be detected timely and thus causes missed chances for surgery. At present, the most commonly and widely used tumor biomarker for early PDAC diagnosis is carbohydrate antigen 19-9 (CA19-9) (Goggins, 2005), but it is not an ideal biomarker because of its relatively low level of sensitivity and specificity (70\% with a 5\% error rate, for diagnosis of PDAC) (Goonetilleke and Siriwardena, 2007; Datta and Vollmer, 2014). Therefore, a reliable signature with exquisitely high sensitivity and specificity is urgently needed to facilitate early PDAC diagnosis.

The main shortcoming of the existing diagnostic signatures is that they are basically obtained by using signature genes' absolute expression value (Klett et al., 2018; Liao et al., 2018; Lu et al., 2018; Cheng et al., 2019b; Zou and Ma, 2020). Therefore, the batch effects could influence the choice of diagnostic signatures. Luckily, we could obtain diagnostic signatures with qualitative transcriptional information through exploiting the relative expression ordering (REO) method. The REO method is highly robust to experimental batch effects (Eddy et al., 2010; Cai et al., 2015; Zhao et al., 2016) and platform differences (Guan et al., 2016; Cheng, 2019). Therefore, it is possible to find robust and reliable disease signatures by using the datasets integrated from different platforms. Moreover, the REO strategy has been successfully used to identify the early diagnosis signature of malignant carcinoma, such as gastric cancer (Yan et al., 2019), hepatocellular carcinoma (Ao et al.,

Abbreviations: PDAC, pancreatic ductal adenocarcinoma; REOs, relative expression orderings; $\mathrm{mRMR}$, maximum relevance minimum redundancy; IFS, incremental feature selection; SVM, support vector machine.
2018), and colorectal cancer (Guan et al., 2019). Consequently, it is worth employing the within-sample REO method to develop a robust qualitative signature for diagnosing earlystage PDAC.

Machine-learning techniques, which can be used to uncover biological principles and mechanism, is a good choice for biological knowledge mining (Liu et al., 2013, 2019; Cao et al., 2017; Cheng et al., 2018a,b; Du et al., 2018; Zou et al., 2018; Stephenson et al., 2019). Hence, this work was devoted to develop an artificial intelligence-based approach to identify early-stage PDAC diagnostic signature. In the first step, all REOs were used for initial diagnosis descriptor. Subsequently, the minimum redundancy maximum relevance (mRMR), a features selection technique, was utilized to remove redundant REOs. The support vector machine (SVM), decision tree, logistic regression, random forest, naïve Bayes, and Bayes net algorithms were used for classification. Finally, 9 salient and genuine gene pairs including 16 genes were screened as the diagnostic signature for diagnosing early-stage PDAC. The nine gene pairs' signature displayed good diagnosis performance for early-stage PDAC in different diagnosis platforms by combining with SVM.

\section{MATERIALS AND METHODS}

\section{The Construction of Datasets}

The microarray gene expression data and RNA-seq data used in current paper were collected from two

TABLE 1 | Statistics of all data sets.

\begin{tabular}{|c|c|c|c|c|c|}
\hline Data set & Platform & PDAC & PDAC_adjacent & Pancreatitis & Normal \\
\hline GSE62452 & Affymetrix GPL6244 & 69 & 61 & - & - \\
\hline GSE28735 & Affymetrix GPL6244 & 45 & 45 & - & - \\
\hline GSE22780 & Affymetrix GPL570 & 8 & 8 & - & - \\
\hline GSE15471 & Affymetrix GPL570 & 39 & 39 & - & - \\
\hline GSE50827 & Illumina GPL10558 & 103 & - & - & - \\
\hline GSE106189 & Affymetrix GPL570 & 35 & - & - & - \\
\hline GSE84219 & Illumina GPL14951 & 30 & - & - & - \\
\hline GSE98399 & Affymetrix GPL570 & 43 & - & - & - \\
\hline GSE62165 & Affymetrix GPL13667 & 118 & - & - & 13 \\
\hline GSE32676 & Affymetrix GPL570 & 25 & - & - & 7 \\
\hline GSE101462 & Illumina GPL10558 & 6 & - & 10 & 4 \\
\hline GSE101448 & Illumina GPL10558 & 24 & - & - & 19 \\
\hline GSE41368 & Affymetrix GPL6244 & 6 & - & - & 6 \\
\hline GSE60601 & Affymetrix GPL570 & 9 & - & - & 3 \\
\hline GSE71989 & Affymetrix GPL570 & 13 & - & - & 8 \\
\hline GSE89120 & Affymetrix GPL1352 & - & - & - & 14 \\
\hline Total & & 573 & 153 & 10 & 74 \\
\hline \multicolumn{6}{|c|}{ Samples for assessing the efficiency of the signature } \\
\hline TCGA & RNA-Seq & 177 & 4 & - & - \\
\hline Total & & 177 & 4 & & \\
\hline
\end{tabular}

PDAC, pancreatic ductal adenocarcinoma tissues; PDAC_adjacent, PDAC adjacent normal tissues. 


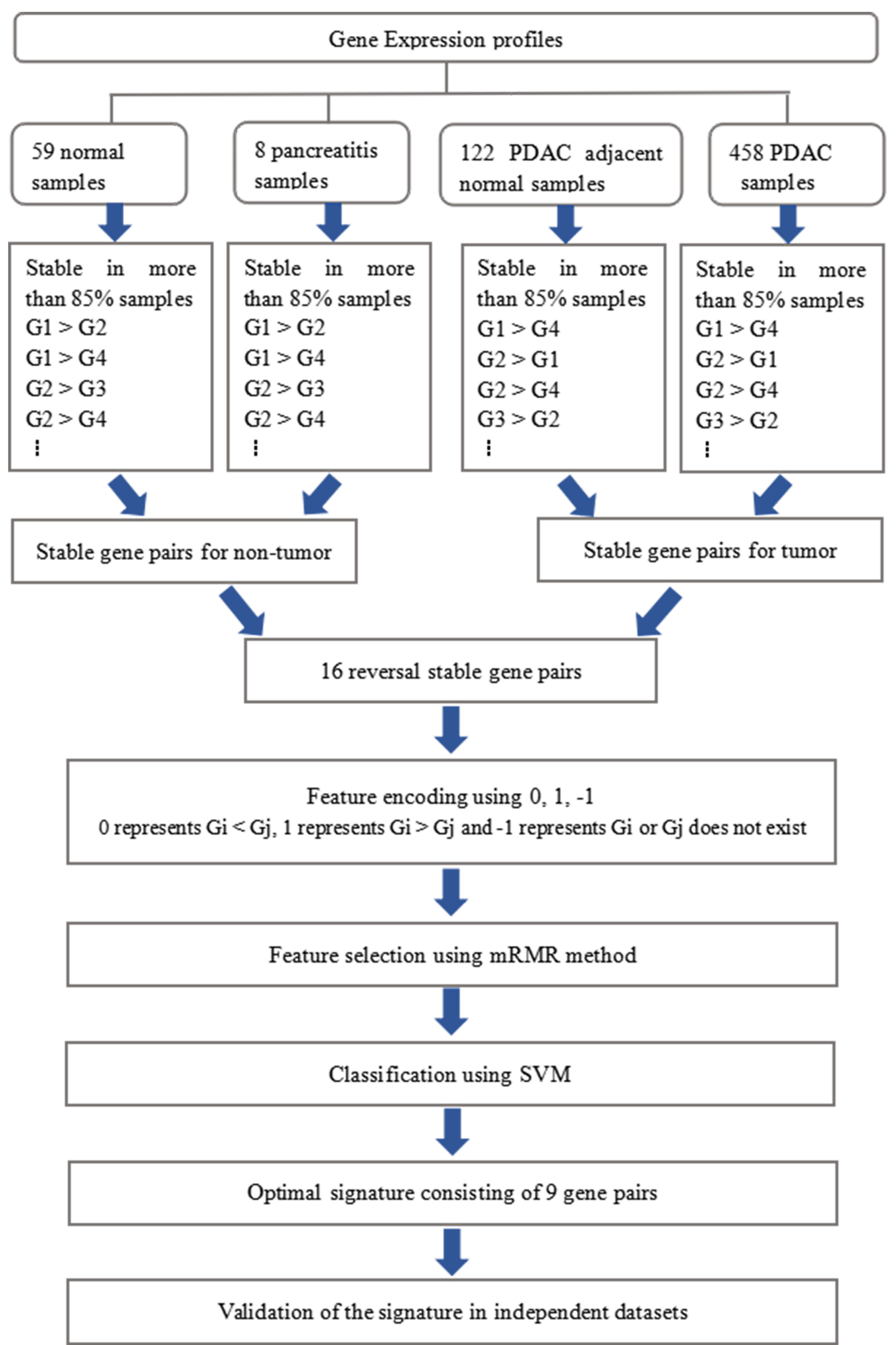

FIGURE 1 | Schematic workflow of analyses. 
TABLE 2 | Comparison of different methods for identifying early PDAC diagnostic biomarker.

\begin{tabular}{|c|c|c|c|c|c|c|c|c|}
\hline \multirow[t]{2}{*}{ Methods } & \multicolumn{4}{|c|}{ Training set } & \multicolumn{4}{|c|}{ Testing set } \\
\hline & ACR (\%) & SES (\%) & SPF (\%) & MCC & ACR (\%) & SES (\%) & SPF (\%) & MCC \\
\hline SVM & 97.53 & 97.96 & 93.22 & 0.8615 & 98.77 & 98.65 & 100.00 & 0.9330 \\
\hline Decision tree & 96.91 & 97.78 & 88.52 & 0.8278 & 95.09 & 97.92 & 73.68 & 0.7518 \\
\hline Logistic regression & 96.91 & 98.11 & 86.15 & 0.8314 & 96.93 & 99.30 & 80.00 & 0.8513 \\
\hline Random forest & 96.60 & 97.61 & 86.89 & 0.8104 & 96.93 & 99.30 & 80.00 & 0.8513 \\
\hline Naïve Bayes & 96.14 & 98.94 & 76.25 & 0.8124 & 96.32 & 99.30 & 76.19 & 0.8274 \\
\hline Bayes net & 95.83 & 98.59 & 75.64 & 0.7933 & 95.70 & 99.29 & 72.73 & 0.8051 \\
\hline
\end{tabular}

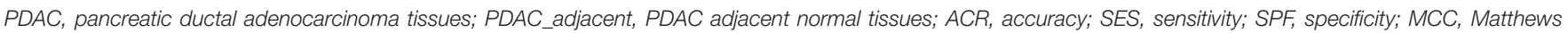
correlation coefficient.

databases: the GEO database ${ }^{1}$ and TCGA database ${ }^{2}$. The detailed description of all data sets is elucidated in Table 1.

Microarray data performed by the platform of Affymetrix and Illumina were freely downloaded from the GEO database. It contained 573 PDAC samples (Set1), 153 PDAC adjacent normal samples (Set2), 10 pancreatitis samples (Set3), and 74 normal samples (Set4). For data performed by Affymetrix, the raw data were directly downloaded from GEO and then the robust multi-array averaging (Bolstad et al., 2003; Irizarry et al., 2003a,b) was used to do the background correction and normalization. For data performed by Illumina, the originally processed data (series matrix files) were used. For all microarray data, the mapping information of probe IDs and Entrez gene IDs can be found in the corresponding platform files. For one gene with multiple probes, we used the arithmetic mean of these probes' values as this gene's expression value.

The RNA-Seq data set included 177 PDAC and 4 adjacent normal samples. We downloaded the free RNA-Seq profiles from TCGA (up to November 19, 2019) website using the TCGAbiolinks $\mathrm{R}$ package (Colaprico et al., 2016). The gene symbol expression matrix was obtained by using Ensembl gene IDs.

${ }^{1}$ http://www.ncbi.nlm.nih.gov/geo/

${ }^{2}$ https://portal.gdc.cancer.gov/



FIGURE 2 | A plot to show the IFS curve. The black dotted line showed that nine gene pairs reached the highest accuracy of $97.53 \%$.
To make the evaluation of the model more objective, each category of samples (Set1, Set2, Set3, Set4) were divided into two subsets of data: training set $(80 \%$ of each category of samples) and testing set $(20 \%$ of each category of samples). Ultimately, the training set contained 580 tumor samples (458 PDAC samples and 122 PDAC adjacent normal samples) and 67 non-tumor samples (59 normal samples and 8 pancreatitis samples). The testing set contained 146 tumor samples (115 PDAC samples and 31 PDAC adjacent normal samples) and 17 nontumor samples (15 normal samples and 2 pancreatitis samples) for the performance evaluation of the signature. In addition, the RNA-Seq data set and testing set belong to the independent test data sets.

\section{Relative Expression Orderings (REOs)}

To obtain a more robust and reliable signature from gene expression profiles, REO methodology was utilized for feature construction. The REO for gene pair ( $i$ and $j$ ) is formulated as $\mathrm{Gi}>\mathrm{Gj}$ or $\mathrm{Gi}<\mathrm{Gj}$, where $\mathrm{Gi}$ and $\mathrm{Gj}$ represent the expression values of gene $\mathrm{i}$ and $\mathrm{j}$. For the gene pair, if more than $85 \%$ of the samples have the same REO, we deem this REO as a stable REO of this gene pair. The stable reversal gene pairs represent the gene pairs that have stable REOs in both tumor tissues and control tissues, but REO patterns are different $(\mathrm{Gi}>\mathrm{Gj}$ or $\mathrm{Gi}<\mathrm{Gj}$ in tumor tissues but $\mathrm{Gi}>\mathrm{Gj}$ or $\mathrm{Gi}<\mathrm{Gj}$ in non-tumor tissues). Also, the reversal stable gene pairs between tumor and control samples were chosen as the candidate REO-based qualitative diagnostic signature. We later gained the consistent genes of all preprocessed data sets and its corresponding gene expression profiles. Whereafter, based on the reversal gene pairs and gene expression profiles, we gained new profiles by using 0,1 , and -1 to denote $\mathrm{Gi}>\mathrm{Gj}, \mathrm{Gi}<\mathrm{Gj}$, and other cases (Gi or $\mathrm{Gj}$ does not exist), respectively.

\section{Minimum Redundancy Maximum Relevance (mRMR)}

The mRMR (Peng et al., 2005) approach can omit the redundant features and choose the high-relevancy features to the target class, and thus significantly improve the classification accuracy. mRMR is on the base of information theory and it can be 
TABLE 3 | The nine gene pairs' signature ranked by mRMR.

\begin{tabular}{lcc}
\hline Order & \multicolumn{2}{c}{ Feature (gene pair) } \\
\cline { 2 - 3 } & Gene $\mathbf{~}$ & Gene j \\
\hline 1 & UBE2C & FITM1 \\
2 & SERPINB5 & ZNF100 \\
3 & NUSAP1 & ONECUT1 \\
4 & LAMC2 & RBM33 \\
5 & BCAR3 & FBXO42 \\
6 & CTSE & PRRC2C \\
7 & HOXB7 & MYO19 \\
8 & NUSAP1 & TNKS \\
9 & RRM2 & ONECUT1 \\
\hline
\end{tabular}

The absolute expression value of gene $i$ is higher than that of gene $j$ in PDAC patients compared with non-PDAC patients.

accomplished through mutual information (MI) operation, and the MI is formulated as follows:

$$
M I\left(v_{i}, C\right)=\int p\left(v_{i}, C\right) \ln \left(\frac{\mathrm{p}\left(\mathrm{v}_{\mathrm{i}}, \mathrm{C}\right)}{\mathrm{p}\left(\mathrm{v}_{\mathrm{i}}\right) \mathrm{p}(\mathrm{C})}\right) d \mathrm{v}_{\mathrm{i}} d \mathrm{C}
$$

where $v$ represents the feature vector and $C$ represents the class to be targeted.

The mRMR is estimated as

$$
m R M R=\frac{1}{|\Psi|} \sum_{v_{i} \in \Psi} M I\left(v_{i}, C\right)-\frac{1}{|\Psi|^{2}} \sum_{v_{i} v_{j} \in \Psi} M I\left(v_{i}, v_{j}\right)
$$

where $\Psi$ denotes the set of ranked features, MI $\left(v_{i}, C\right)$ denotes mutual information between the $v_{i}$ feature and class C, and IM $\left(v_{i}, v_{j}\right)$ denotes mutual information between $v_{i}$ and $v_{j}$.

In this work, a reversal stable gene pair was considered as a feature. The feature selection process was essential for exact classification between tumor samples (positive samples) and non-tumor samples (negative samples). Thus, we utilized mRMR method to pick out effective features (gene pairs).

\section{Incremental Feature Selection (IFS)}

Based on mRMR techniques, we gained a list of ranked features (gene pairs). The incremental feature selection (IFS) (Li et al., 2019) strategy was adopted to find the optimal feature subset which could produce the best diagnosis for PDAC. During IFS process, the gene pair was added one by one to feature subset and the optimal features (gene pairs) were determined when the highest accuracy was obtained.

\section{Classification Algorithms}

As a popular supervised learning approach, SVM was first introduced by Vapnik and has been widely used in various bioinformatics classification problems (Song et al., 2009; Shoombuatong et al., 2012; Win et al., 2017, 2018; Chen et al., 2019b; Laengsri et al., 2019; Manavalan et al., 2019b; Schaduangrat et al., 2019; Hasan et al., 2020; Liu and Chen, 2020). Herein, the free LibSVM (version 3.23) package
(Chang and Lin, 2011) was employed to execute SVM. The LibSVM with fivefold cross-validation and radial basis function was employed to perform classification. The grid search with fivefold cross-validation was used to determine the $C$ and $\gamma$ values for SVM. As a result, we obtained the optimal values 32 and 0.03125 for $C$ and $\gamma$, respectively. Apart from SVM, decision tree, logistic regression, random forest, naïve Bayes, and Bayes net were also utilized as classification algorithm and performed by using Weka (version 3.8.3) (Frank et al., 2004). Within this research, the aforementioned six classification algorithms with fivefold cross-validation were used.

\section{Performance Measurements}

In the current paper, six indexes were used to measure the effectiveness of our model. They are accuracy (ACR), sensitivity (SES), specificity (SPF), Matthews correlation coefficient (MCC) (Li et al., 2015; Bao et al., 2019; Chen et al., 2019a; Cheng et al., 2019a, 2020), the receiver operating characteristic (ROC) curves, and the area under the ROC curve (AUC). Especially, taking into consideration the class imbalance of tumor tissues and non-tumor tissues, we appointed MCC as the major performance measurement in this work. The details about ACR, SES, SPF, and MCC can be found from Tang et al. (2017); Basith et al. (2019), Manavalan et al. (2019a); Patil and Chouhan (2019), and Basith et al. (2020).

\section{RESULTS}

\section{Derivation of PDAC Diagnostic Signature}

The whole procedure of deriving the diagnostic signature is provided in Figure 1. First, with the relative expression orderings elaborated in Materials and Methods section, for 458 PDAC samples and 122 PDAC adjacent normal samples in the training set, there were $30,865,512$ and $49,177,748$ stable gene pairs, respectively. Also, there were 17,842,291 consistent stable gene pairs in total. Likewise, for 8 pancreatitis samples and 59 normal samples in the training set, there were $53,719,117$ and $44,523,890$ stable gene pairs, respectively. There were 25,687,362 consistent stable gene pairs in total. Among 17,842,291 and 25,687,362 gene pairs, there were 16 stable reversal gene pairs between the two sets of samples. Then, on the basis of the novel profiles (see Materials and Methods), we captured the optimal feature set from the 16 gene pairs by using mRMR with SVM, decision tree, logistic regression, random forest, naïve Bayes, and Bayes net. The comparison results of the aforementioned six classification algorithms are listed in Table 2. It was obvious that the SVM algorithm was the best one for identifying early PDAC diagnostic biomarker. The accuracy, sensitivity, specificity, and MCC of SVM were, respectively, 97.53\%, 97.96\%, 93.22\% and 0.8615 . Therefore, the final model used for early PDAC diagnostic biomarker identification was built based on SVM algorithm. The blue curve in Figure 2 displayed the process of IFS method. As we could see from Figure 2, with fivefold cross-validation, the nine gene pair 
TABLE 4 | Classification efficiency of the nine gene pairs in independent test data sets.

\begin{tabular}{|c|c|c|c|c|c|c|c|c|}
\hline Data set & PDAC & PDAC_adjacent & Pancreatitis & Normal & ACR & SES & SPF & MCC \\
\hline Testing set & 115 & 31 & 2 & 15 & $98.77 \%$ & $98.65 \%$ & $100.00 \%$ & 0.9330 \\
\hline TCGA & 177 & 4 & - & - & - & $100.00 \%$ & - & - \\
\hline
\end{tabular}


correlation coefficient.

signature could identify PDAC with up to $97.53 \%$ accuracy on training set. That is to say, nine gene pairs illustrated in Table 3 were deemed as the optimal signature for diagnosing the earlystage PDAC.

\section{Examination of the Signature}

We then assessed the classification ability of nine gene pairs in independent test data sets, and the test results with fivefold cross-validation are shown in Table 4. For 163 samples in the testing set, our model reached accuracy, sensitivity, specificity, and MCC values of $98.77 \%$, 98.65\%, $100.00 \%$, and 0.9330, respectively. Furthermore, the signature 9 gene pairs could accurately distinguish 177 PDAC samples and 4 PDAC adjacent normal samples measured by RNA-Seq although the training set did not contain any RNA-Seq information. This test result, based on RNA-Seq data set, indicated that the nine gene pairs have a good cross-platform effect for PDAC early detection. For all 327 PDAC samples and 17 nonPDAC samples collected from public databases, the accuracy, sensitivity, specificity, and MCC are 99.42\%, 99.39\%, 100\%, and 0.9365, respectively. Also, the AUC reached 0.9524 (95\% CI, 0.8881-1; see Figure 3). According to independent tests on testing set and RNA-Seq data set, it was concluded that the signature could discriminate PDAC (PDAC and adjacent normal tissues) patients from non-PDAC (pancreatitis and normal tissues) patients.

\section{DISCUSSION}

Pancreatic carcinoma is a life-threatening malignant tumor of the digestive system with bad prognosis due to late diagnosis. The current imaging techniques and existing tumor signatures have insufficient sensitivity and/or specificity for early PDAC diagnosis. Herein, new strategies for diagnosis at an early stage of the disease are urgently needed. In the current work, we found a robust qualitative diagnostic signature 9 gene pairs (16 genes), which can discriminate PDAC (PDAC and adjacent normal tissues) patients from non-PDAC (pancreatitis and normal tissues) and might be a promising biomarker for early diagnosis of PDAC.

Database PubMed was searched and retrieved appropriate journal articles on the association between 16 genes in 9 gene pairs and PDAC published before August 18, 2020. Seven genes in the nine gene pairs' signature, including UBE2C, SERPINB5, LAMC2, CTSE, HOXB7, RRM2, and ONECUT1, had been reported to be related to PDAC. The description of the association between seven genes and PDAC is displayed in Table 5. They might play a vital role in PDAC tumorigenesis and were critical genes for cancer. Notably, CTSE (Keliher et al., 2013), HOXB7 (Chile et al., 2013; Nguyen Kovochich et al., 2013), and RRM2 (Bhutia et al., 2013) were overexpressed in PDAC. UBE2C could encode a ubiquitin-conjugating enzyme which correlated with the PDAC development and progression. Also, the proliferation and epithelial-mesenchymal transition in PDAC could be inhibited by silencing UBE2C (Wang et al., 2019). SERPINB5 had been found to link to the prognosis of PDAC (Cheng et al., 2019b). LAMC2 has relation with the occurrence and progression of PDAC patients (Pan et al., 2018; Yang et al., 2018; Zhang et al., 2018a). Furthermore, the high expression level of LAMC2 could facilitate the invasion of PDAC cell and thus increase the risk of tumor recurrence (Yang et al., 2018). Patients with pancreatic diseases (chronic pancreatitis) had a higher risk of developing PDAC and thus the expression of CTSE in pancreatic diseases might be the key to early PDAC detection and PDAC progression. HOXB7, frequently overexpressed in PDAC, closely connected with lymph node metastasis (Nguyen Kovochich et al., 2013) and worse survival in PDAC patients (Zhang et al., 2014). Knockdowning HOXB7 could cause cell apoptosis and cell cycle arrest (Chile et al., 2013). RRM2

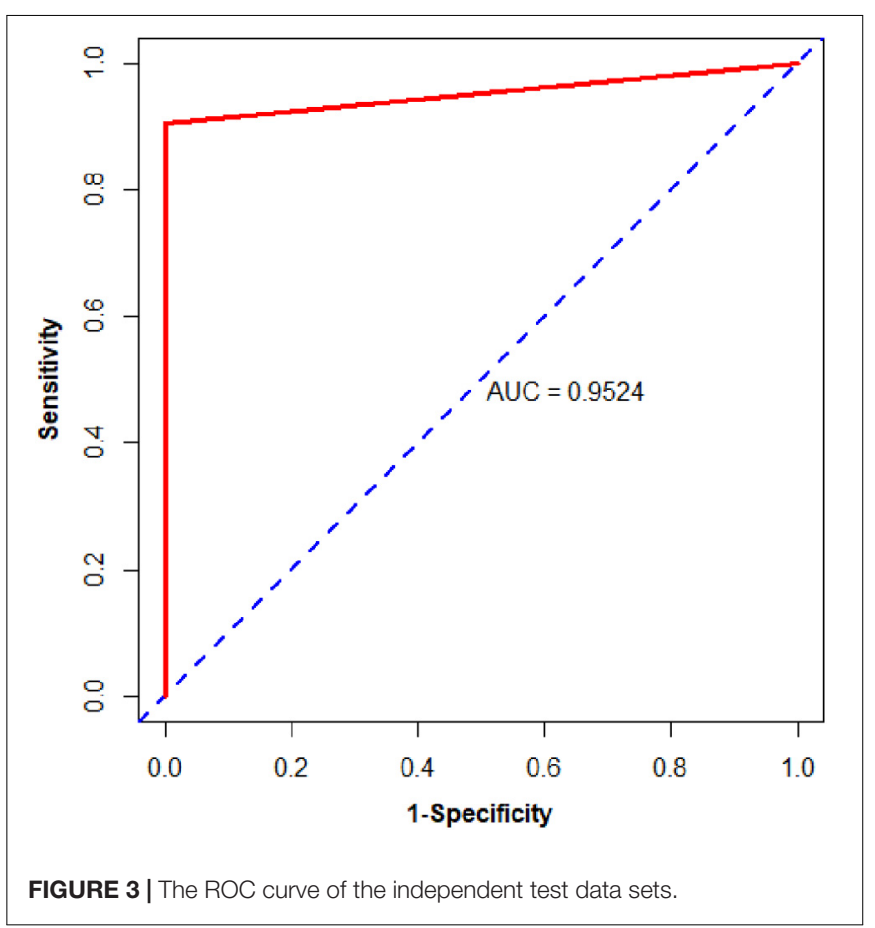


TABLE 5 | The description of the association between seven genes and PDAC.

\begin{tabular}{|c|c|}
\hline Gene symbol & $\begin{array}{l}\text { The description of the } \\
\text { association between } \\
\text { seven genes and PDAC }\end{array}$ \\
\hline UBE2C & $\begin{array}{l}\text { Silencing UBE2C could } \\
\text { inhibit the proliferation and } \\
\text { epithelial-mesenchymal } \\
\text { transition in PDAC } \\
\text { (Manavalan et al., 2019a) }\end{array}$ \\
\hline SERPINB5 & $\begin{array}{l}\text { SERPINB5 links to the } \\
\text { prognosis of PDAC (Cheng } \\
\text { et al., 2019b) }\end{array}$ \\
\hline LAMC2 & $\begin{array}{l}\text { LAMC2 is associated with } \\
\text { PDAC occurrence and } \\
\text { progression (54-56). The } \\
\text { high expression level of } \\
\text { LAMC2 could facilitate the } \\
\text { invasion of PDAC cell and } \\
\text { thus increase the risk of } \\
\text { tumor recurrence (Keliher } \\
\text { et al., 2013) }\end{array}$ \\
\hline CTSE & $\begin{array}{l}\text { Because patients with } \\
\text { pancreatic diseases } \\
\text { (chronic pancreatitis) have a } \\
\text { strong risk of developing } \\
\text { PDAC, the expression of } \\
\text { CTSE in pancreatic } \\
\text { diseases might be the key } \\
\text { to detection of early PDAC } \\
\text { and progression of PDAC }\end{array}$ \\
\hline HOXB7 & $\begin{array}{l}\text { HOXB7 is overexpressed in } \\
\text { PDAC. It is closely relevant } \\
\text { to lymph node metastasis } \\
\text { (Patil and Chouhan, 2019) } \\
\text { and worse survival of PDAC } \\
\text { patients (Chile et al., 2013). } \\
\text { Knockdowning HOXB7 can } \\
\text { cause cell apoptosis and } \\
\text { cell cycle arrest (Tang et al., } \\
\text { 2017) }\end{array}$ \\
\hline RRM2 & $\begin{array}{l}\text { Gene expression of RRM2 } \\
\text { was significantly higher in } \\
\text { PDAC tissues than normal } \\
\text { pancreatic tissues (Basith } \\
\text { et al., 2019) }\end{array}$ \\
\hline ONECUT1 & $\begin{array}{l}\text { Loss expression of } \\
\text { ONECUT1 in PDAC cells } \\
\text { implied its tumor } \\
\text { suppressor function in this } \\
\text { malignant tumor (Wang } \\
\text { et al., 2019) }\end{array}$ \\
\hline
\end{tabular}

was involved in the process of deoxyribonucleotide synthesis. Gene expression of RRM2 was significantly higher in PDAC tissues than in normal pancreatic tissues, which brought about the chemoresistance of PDAC to nucleoside analogs (Bhutia et al., 2013). A loss of ONECUT1 expression in PDAC cells implied its tumor suppressor function in this malignant tumor (Jiang et al., 2008).

To further study the detailed information and functions of the 9 gene pairs, we analyzed 16 genes ( 9 gene pairs) via using online tools in Metascape ${ }^{3}$ (Tripathi et al., 2015). The enrichment analysis included GO terms functional enrichment and KEGG pathway enrichment. Pathways with $P$-value were less than 0.05 and the number of enriched genes greater than or equal to 3 was considered significant. Ultimately, based on the GO enrichment, the 16 genes ( 9 gene pairs) enriched in two terms in the category BP, including "regulation of cell cycle process" and "regulation of mitotic nuclear division." UBE2C, RRM2, TNKS, NUSAP1, and MYO19 were included in the genes enriched in regulation of cell cycle process, whereas TNKS, UBE2C, NUSAP1, and MYO19 enriched in the regulation of mitotic nuclear division. Collecting the aforementioned results, the genes of the nine gene pairs might play a significant part in the tumorigenesis of PDAC.

In conclusion, we had identified nine gene pairs' signature for early-stage PDAC diagnosis that could correctly distinguish PDAC (PDAC and PDAC adjacent normal tissues) tissues from non-PDAC (normal and pancreatitis tissues) patients at individual level. Because the number of normal and pancreatitis samples used in the current work for distinguishing early-stage PDAC is relatively small, we will try to collect more samples from more public databases to further obtain a novel diagnostic signature with higher accuracy on larger numbers of such specimens. Moreover, we hope that some RNA signature (Fang et al., 2019; Vaschetto, 2019; Wu et al., 2019) can be found and applied in related fields.

\section{DATA AVAILABILITY STATEMENT}

Publicly available datasets were analyzed in this study. This data can be found here: https://portal.gdc.cancer.gov/ and http://www. ncbi.nlm.nih.gov/geo/.

\section{AUTHOR CONTRIBUTIONS}

HL designed and supervised the study. Z-MZ collected all datasets and wrote the article with the help of HL and F-YD. Z-MZ, J-SW, HZ, HL, and F-YD performed the experiments. All authors contributed to the article and approved the submitted version.

\section{FUNDING}

This research project was supported by the National Natural Scientific Foundation of China (61772119) and the Sichuan Provincial Science Fund for Distinguished Young Scholars (2020JDJQ0012).

\section{ACKNOWLEDGMENTS}

We thank all colleagues in our laboratory for their generous suggestions and support.

\footnotetext{
${ }^{3}$ http://metascape.org
} 


\section{REFERENCES}

Ao, L., Zhang, Z., Guan, Q., Guo, Y., Guo, Y., Zhang, J., et al. (2018). A qualitative signature for early diagnosis of hepatocellular carcinoma based on relative expression orderings. Liver Int. 38, 1812-1819. doi: 10.1111/liv.13864

Bao, Y., Marini, S., Tamura, T., Kamada, M., Maegawa, S., Hosokawa, H., et al. (2019). Toward more accurate prediction of caspase cleavage sites: a comprehensive review of current methods, tools and features. Brief. Bioinform. 20, 1669-1684. doi: 10.1093/bib/bby041

Basith, S., Manavalan, B., Shin, T. H., and Lee, G. (2019). SDM6A: a web-based integrative machine-learning framework for predicting $6 \mathrm{~mA}$ sites in the rice genome. Mol. Ther. Nucleic Acids 18, 131-141. doi: 10.1016/j.omtn.2019.08.011

Basith, S., Manavalan, B., Shin, T. H., and Lee, G. (2020). Machine intelligence in peptide therapeutics: a next-generation tool for rapid disease screening. Med. Res. Rev. 40, 1276-1314. doi: 10.1002/med.21658

Bhutia, Y. D., Hung, S. W., Krentz, M., Patel, D., Lovin, D., Manoharan, R., et al. (2013). Differential processing of let-7a precursors influences RRM2 expression and chemosensitivity in pancreatic cancer: role of LIN-28 and SET oncoprotein. PLoS One 8:e53436. doi: 10.1371/journal.pone.0053436

Bolstad, B. M., Irizarry, R. A., Astrand, M., and Speed, T. P. (2003). A comparison of normalization methods for high density oligonucleotide array data based on variance and bias. Bioinformatics 19, 185-193. doi: 10.1093/bioinformatics/19. 2.185

Cai, H., Li, X., Li, J., Ao, L., Yan, H., Tong, M., et al. (2015). Tamoxifen therapy benefit predictive signature coupled with prognostic signature of post-operative recurrent risk for early stage ER+ breast cancer. Oncotarget 6, 44593-44608. doi: $10.18632 /$ oncotarget. 6260

Cao, R., Freitas, C., Chan, L., Sun, M., Jiang, H., and Chen, Z. (2017). ProLanGO: protein function prediction using neural machine translation based on a recurrent neural network. Molecules 22:1732. doi: 10.3390/molecules22101732

Chang, C. C., and Lin, C. J. (2011). LIBSVM: a library for support vector machines. ACM T Intel. Syst. Tec. 2, 1-27. doi: 10.1145/1961189.1961199

Chen, W., Feng, P., Liu, T., and Jin, D. (2019a). Recent advances in machine learning methods for predicting heat shock proteins. Curr. Drug Metab 20, 224-228. doi: 10.2174/1389200219666181031105916

Chen, W., Feng, P., and Nie, F. (2019b). iATP: a sequence based method for identifying anti-tubercular peptides. Med. Chem. 16, 620-625. doi: 10.2174/ 1573406415666191002152441

Cheng, L. (2019). Computational and biological methods for gene therapy. Curr. Gene Ther. 19:210. doi: 10.2174/156652321904191022113307

Cheng, L., Hu, Y., Sun, J., Zhou, M., and Jiang, Q. (2018a). DincRNA: a comprehensive web-based bioinformatics toolkit for exploring disease associations and ncRNA function. Bioinformatics 34, 1953-1956. doi: 10.1093/ bioinformatics/bty002

Cheng, L., Jiang, Y., Ju, H., Sun, J., Peng, J., Zhou, M., et al. (2018b). InfAcrOnt: calculating cross-ontology term similarities using information flow by a random walk. BMC Genomics 19:919. doi: 10.1186/s12864-017-4338-6

Cheng, L., Qi, C., Zhuang, H., Fu, T., and Zhang, X. (2020). gutMDisorder: a comprehensive database for dysbiosis of the gut microbiota in disorders and interventions. Nucleic Acids Res. 48, D554-D560.

Cheng, L., Zhao, H., Wang, P., Zhou, W., Luo, M., Li, T., et al. (2019a). Computational methods for identifying similar diseases. Mol. Ther. Nucleic Acids 18, 590-604. doi: 10.1016/j.omtn.2019.09.019

Cheng, Y., Wang, K., Geng, L., Sun, J., Xu, W., Liu, D., et al. (2019b). Identification of candidate diagnostic and prognostic biomarkers for pancreatic carcinoma. EBIO Med. 40, 382-393. doi: 10.1016/j.ebiom.2019.01.003

Chile, T., Fortes, M. A., Correa-Giannella, M. L., Brentani, H. P., Maria, D. A., Puga, R. D., et al. (2013). HOXB7 mRNA is overexpressed in pancreatic ductal adenocarcinomas and its knockdown induces cell cycle arrest and apoptosis. BMC Cancer 13:451. doi: 10.1186/1471-2407-13-451

Colaprico, A., Silva, T. C., Olsen, C., Garofano, L., Cava, C., Garolini, D., et al. (2016). TCGAbiolinks: an R/Bioconductor package for integrative analysis of TCGA data. Nucleic Acids Res. 44:e71. doi: 10.1093/nar/gkv1507

Datta, J., and Vollmer, C. M., Jr. (2014). Investigational biomarkers for pancreatic adenocarcinoma: where do we stand? Southern Med. J. 107, 256-263. doi: 10.1097/smj.0000000000000088

Du, X. Q., Li, X. R., Li, W., Yan, Y. T., and Zhang, Y. P. (2018). Identification and analysis of cancer diagnosis using probabilistic classification vector machines with feature selection. Curr. Bioinform. 13, 625-632. doi: 10.2174/ 1574893612666170405125637

Eddy, J. A., Sung, J., Geman, D., and Price, N. D. (2010). Relative expression analysis for molecular cancer diagnosis and prognosis. Technol. Cancer Res. Treatment 9, 149-159. doi: 10.1177/153303461000900204

Fang, S., Pan, J. C., Zhou, C. W., Tian, H., He, J. X., Shen, W. Y., et al. (2019). Circular RNAs serve as novel biomarkers and therapeutic targets in cancers. Curr. Gene Ther. 19, 125-133. doi: 10.2174/1566523218666181109142756

Frank, E., Hall, M., Trigg, L., Holmes, G., and Witten, I. H. (2004). Data mining in bioinformatics using weka. Bioinformatics 20, 2479-2481. doi: 10.1093/ bioinformatics/bth261

Goggins, M. (2005). Molecular markers of early pancreatic cancer. J. Clin. Oncol. 23, 4524-4531. doi: 10.1200/jco.2005.19.711

Goonetilleke, K. S., and Siriwardena, A. K. (2007). Systematic review of carbohydrate antigen (CA 19-9) as a biochemical marker in the diagnosis of pancreatic cancer. Eur. J. Surg. 33, 266-270. doi: 10.1016/j.ejso.2006.10.004

Guan, Q., Chen, R., Yan, H., Cai, H., Guo, Y., Li, M., et al. (2016). Differential expression analysis for individual cancer samples based on robust withinsample relative gene expression orderings across multiple profiling platforms. Oncotarget 7, 68909-68920. doi: 10.18632/oncotarget.11996

Guan, Q., Zeng, Q., Yan, H., Xie, J., Cheng, J., Ao, L., et al. (2019). A qualitative transcriptional signature for the early diagnosis of colorectal cancer. Cancer Sci. 110, 3225-3234. doi: $10.1111 /$ cas. 14137

Hasan, M. M., Schaduangrat, N., Basith, S., Lee, G., Shoombuatong, W., and Manavalan, B. (2020). HLPpred-Fuse: improved and robust prediction of hemolytic peptide and its activity by fusing multiple feature representation. Bioinformatics 36, 3350-3356. doi: 10.1093/bioinformatics/btaal60

Irizarry, R. A., Bolstad, B. M., Collin, F., Cope, L. M., Hobbs, B., and Speed, T. P. (2003a). Summaries of affymetrix genechip probe level data. Nucleic Acids Res. 31:e15.

Irizarry, R. A., Hobbs, B., Collin, F., Beazer-Barclay, Y. D., Antonellis, K. J., Scherf, U., et al. (2003b). Exploration, normalization, and summaries of high density oligonucleotide array probe level data. Biostatistics 4, 249-264. doi: 10.1093/ biostatistics/4.2.249

Jiang, X., Zhang, W., Kayed, H., Zheng, P., Giese, N. A., Friess, H., et al. (2008). Loss of ONECUT1 expression in human pancreatic cancer cells. Oncol. Rep. 19, 157-163.

Keliher, E. J., Reiner, T., Earley, S., Klubnick, J., Tassa, C., Lee, A. J., et al. (2013). Targeting cathepsin $\mathrm{E}$ in pancreatic cancer by a small molecule allows in vivo detection. Neoplasia 15, 684-693. doi: 10.1593/neo.13276

Klett, H., Fuellgraf, H., Levit-Zerdoun, E., Hussung, S., Kowar, S., Kusters, S., et al. (2018). Identification and validation of a diagnostic and prognostic multi-gene biomarker panel for pancreatic ductal adenocarcinoma. Front. Genet. 9:108. doi: 10.3389/fgene.2018.00108

Laengsri, V., Nantasenamat, C., Schaduangrat, N., Nuchnoi, P., Prachayasittikul, V., and Shoombuatong, W. (2019). TargetAntiAngio: a sequence-based tool for the prediction and analysis of anti-angiogenic peptides. Int. J. Mol. Sci. 20:2950. doi: 10.3390/ijms 20122950

Li, F., Li, C., Wang, M., Webb, G. I., Zhang, Y., Whisstock, J. C., et al. (2015). GlycoMine: a machine learning-based approach for predicting $\mathrm{N}$-, C- and O-linked glycosylation in the human proteome. Bioinformatics 31, 1411-1419. doi: 10.1093/bioinformatics/btu852

Li, S. H., Zhang, J., Zhao, Y. W., Dad, F. Y., Ding, H., Chen, W., et al. (2019). iPhoPred: a predictor for identifying phosphorylation sites in human protein. IEEE Access. 7, 177517-177528. doi: 10.1109/access.2019.2953951

Liao, Z. J., Li, D. P., Wang, X. R., Li, L. S., and Zou, Q. (2018). Cancer diagnosis through IsomiR expression with machine learning method. Curr. Bioinform. 13, 57-63. doi: 10.2174/1574893611666160609081155

Liu, B., Han, L., Liu, X., Wu, J., and Ma, Q. (2019). Computational prediction of Sigma-54 promoters in bacterial genomes by integrating motif finding and machine learning strategies. IEEE/ACM Trans. Comput. Biol. Bioinform. 16, 1211-1218. doi: $10.1109 / \mathrm{tcbb} .2018 .2816032$

Liu, K., and Chen, W. (2020). iMRM: a platform for simultaneously identifying multiple kinds of RNA modifications. Bioinformatics 36, 3336-3342. doi: 10. 1093/bioinformatics/btaa155

Liu, Y., Guo, J., Hu, G., and Zhu, H. (2013). Gene prediction in metagenomic fragments based on the SVM algorithm. BMC Bioinformatics 14(Suppl. 5):S12. doi: 10.1186/1471-2105-14-S5-S12 
Lu, Y., Li, C., Chen, H., and Zhong, W. (2018). Identification of hub genes and analysis of prognostic values in pancreatic ductal adenocarcinoma by integrated bioinformatics methods. Mol. Biol. Rep. 45, 1799-1807. doi: 10.1007/s11033018-4325-2

Manavalan, B., Basith, S., Shin, T. H., Wei, L., and Lee, G. (2019a). AtbPpred: a robust sequence-based prediction of anti-tubercular peptides using extremely randomized trees. Comput. Struct. Biotechnol. J. 17, 972-981. doi: 10.1016/j. csbj.2019.06.024

Manavalan, B., Basith, S., Shin, T. H., Wei, L. Y., and Lee, G. (2019b). Meta$4 \mathrm{mCpred}$ : a sequence-based meta-predictor for accurate DNA $4 \mathrm{mC}$ site prediction using effective feature representation. Mol. Ther-Nucl Acids 16, 733-744. doi: 10.1016/j.omtn.2019.04.019

Nguyen Kovochich, A., Arensman, M., Lay, A. R., Rao, N. P., Donahue, T., Li, X., et al. (2013). HOXB7 promotes invasion and predicts survival in pancreatic adenocarcinoma. Cancer 119, 529-539. doi: 10.1002/cncr.27725

Pan, Z., Li, L., Fang, Q., Zhang, Y., Hu, X., Qian, Y., et al. (2018). Analysis of dynamic molecular networks for pancreatic ductal adenocarcinoma progression. Cancer cell international 18:214.

Patil, K., and Chouhan, U. (2019). Relevance of machine learning techniques and various protein features in protein fold classification: a review. Curr. Bioinform. 14, 688-697. doi: 10.2174/1574893614666190204154038

Peng, H., Long, F., and Ding, C. (2005). Feature selection based on mutual information: criteria of max-dependency, max-relevance, and min-redundancy. IEEE Trans. Pattern Anal. Mach. Intell. 27, 1226-1238. doi: 10.1109/tpami. 2005.159

Schaduangrat, N., Nantasenamat, C., Prachayasittikul, V., and Shoombuatong, W. (2019). ACPred: a computational tool for the prediction and analysis of anticancer peptides. Molecules 24:1973. doi: 10.3390/molecules24101973

Shoombuatong, W., Hongjaisee, S., Barin, F., Chaijaruwanich, J., and Samleerat, T. (2012). HIV-1 CRF01_AE coreceptor usage prediction using kernel methods based logistic model trees. Comput. Biol. Med. 42, 885-889. doi: 10.1016/j. compbiomed.2012.06.011

Song, J., Tan, H., Mahmood, K., Law, R. H., Buckle, A. M., Webb, G. I., et al. (2009). Prodepth: predict residue depth by support vector regression approach from protein sequences only. PLoS One 4:e7072. doi: 10.1371/journal.pone. 0007072

Stephenson, N., Shane, E., Chase, J., Rowland, J., Ries, D., Justice, N., et al. (2019). Survey of machine learning techniques in drug discovery. Curr. Drug Metab 20, 185-193. doi: 10.2174/1389200219666180820112457

Tanaka, S. (2016). Molecular pathogenesis and targeted therapy of pancreatic cancer. Ann. Surg. Oncol. 23(Suppl. 2), S197-S205.

Tang, H., Cao, R. Z., Wang, W., Liu, T. S., Wang, L. M., and He, C. M. (2017). A two-step discriminated method to identify thermophilic proteins. Int. J. Biomath. 10:1750050. doi: 10.1142/s1793524517 500504

Tripathi, S., Pohl, M. O., Zhou, Y., Rodriguez-Frandsen, A., Wang, G., Stein, D. A., et al. (2015). Meta- and orthogonal integration of influenza "OMICs" data defines a role for UBR4 in virus budding. Cell Host Microbe 18, 723-735. doi: 10.1016/j.chom.2015.11.002

Vaschetto, L. M. (2019). The emergence of non-coding RNAs as versatile and efficient therapeutic tools. Curr. Gene Ther. 19, 289-289. doi: 10.2174/ 156652321905191122154955
Wang, X., Yin, L., Yang, L., Zheng, Y., Liu, S., Yang, J., et al. (2019). Silencing ubiquitin-conjugating enzyme $2 \mathrm{C}$ inhibits proliferation and epithelialmesenchymal transition in pancreatic ductal adenocarcinoma. FEBS J. 286, 4889-4909. doi: 10.1111/febs.15134

Win, T. S., Malik, A. A., Prachayasittikul, V., Wikberg, J. E. S., Nantasenamat, C., and Shoombuatong, W. (2017). HemoPred: a web server for predicting the hemolytic activity of peptides. Future Med. Chem. 9, 275-291. doi: 10.4155/ fmc-2016-0188

Win, T. S., Schaduangrat, N., Prachayasittikul, V., Nantasenamat, C., and Shoombuatong, W. (2018). PAAP: a web server for predicting antihypertensive activity of peptides. Future Med. Chem. 10, 1749-1767. doi: 10.4155/fmc-20170300

Wu, Y. G., Lu, X. X., Shen, B., and Zeng, Y. (2019). The therapeutic potential and role of miRNA, IncRNA, and circRNA in osteoarthritis. Curr. Gene Ther. 19, 255-263. doi: 10.2174/1566523219666190716092203

Yan, H., Li, M., Cao, L., et al. (2019). A robust qualitative transcriptional signature for the correct pathological diagnosis of gastric cancer. J. Trans. Med. 17:63.

Yang, C., Liu, Z., Zeng, X., Wu, Q., Liao, X., Wang, X., et al. (2018). Evaluation of the diagnostic ability of laminin gene family for pancreatic ductal adenocarcinoma. Aging 11, 3679-3703. doi: 10.18632/aging.102007

Zhang, R., Leng, H., Huang, J., Du, Y., Wang, Y., Zang, W., et al. (2014). miR-337 regulates the proliferation and invasion in pancreatic ductal adenocarcinoma by targeting HOXB7. Diagnostic Pathol. 9:171.

Zhang, Y., Zoltan, M., Riquelme, E., Xu, H., Sahin, I., Castro-Pando, S., et al. (2018a). Immune cell production of interleukin 17 induces stem cell features of pancreatic intraepithelial neoplasia cells. Gastroenterology 155, 210-223.e3.

Zhang, Z., Pan, B., Lv, S., Ji, Z., Wu, Q., Lang, R., et al. (2018b). Integrating MicroRNA expression profiling studies to systematically evaluate the diagnostic value of MicroRNAs in pancreatic cancer and validate their prognostic significance with the cancer genome atlas data. Cell. Physiol. Biochem. 49, 678-695. doi: 10.1159/000493033

Zhao, W., Chen, B., Guo, X., Wang, R., Chang, Z., Dong, Y., et al. (2016). A rankbased transcriptional signature for predicting relapse risk of stage II colorectal cancer identified with proper data sources. Oncotarget 7, 19060-19071. doi: 10.18632/oncotarget.7956

Zou, Q., and Ma, Q. (2020). The application of machine learning to disease diagnosis and treatment. Math. Biosci. 320:108305. doi: 10.1016/j.mbs.2019. 108305

Zou, Q., Qu, K., Luo, Y., Yin, D., Ju, Y., and Tang, H. (2018). Predicting diabetes mellitus with machine learning techniques. Front. Genet. 9:515. doi: 10.3389/ fgene.2018.00515

Conflict of Interest: The authors declare that the research was conducted in the absence of any commercial or financial relationships that could be construed as a potential conflict of interest.

Copyright (c) 2020 Zhang, Wang, Zulfiqar, Lv, Dao and Lin. This is an open-access article distributed under the terms of the Creative Commons Attribution License (CC BY). The use, distribution or reproduction in other forums is permitted, provided the original author(s) and the copyright owner(s) are credited and that the original publication in this journal is cited, in accordance with accepted academic practice. No use, distribution or reproduction is permitted which does not comply with these terms. 